\title{
La formación de los profesores de Educación Física en el área de la discapacidad: un análisis de los planes de estudios de 1992, 1996 y 2004 del curso de profesores del ISEF - Montevideo
}

Sofía Rubinstein*

\begin{abstract}
Resumen: Este artículo presenta aspectos relacionados con la formación en el área de la actividad motora adaptada de los profesores de Educación Física, partiendo del estudio de las asignaturas vinculadas a esa área en los tres últimos planes de estudios (1992, 1996 y 2004) del Curso de Profesores del Instituto Superior de Educación Física (ISEF - Montevideo). Los resultados obtenidos muestran un interés en ofrecer por lo menos una asignatura vinculada al área, sin embargo, la fundamentación, objetivos y contenidos se enmarcan en el modelo médico de discapacidad, observándose igualmente un cambio hacia el modelo social en el plan de 2004.
\end{abstract}

Palabras clave: Docentes. Discapacidad. Curso de capacitación. Curriculum

\section{INTRODUCCIÓN}

A través de este artículo se desea contribuir a la construcción de la actividad motora adaptada de nuestro país. En este sentido, como estudiante primero y docente después vinculada a la formación universitaria, siempre existió una preocupación relacionada con la formación que reciben los futuros docentes en el área de la discapacidad. En esta línea, Sosa $(2010$, p. 1) sostiene que "[... ] el problema de la formación de profesionales sobre la temática de la discapacidad excede al campo de la Educación Física"; explica que "[...] las demandas sociales actuales obligan a dar respuesta sobre

"Instituto Universitario Asociación Cristiana de Jóvenes (IUACJ). Montevideo, Uruguay. Email: sofiar@adinet.com.uy 
un sector poblacional excluido, donde se les ha quitado todos sus derechos [...]." La idea de la autora muestra cómo el tema de la discapacidad va más allá de un área del conocimiento en particular y debería estar contemplada en todos los niveles educativos, incluso en los diferentes ámbitos que componen la sociedad.

Se entiende por actividad motora adaptada como un término que engloba los ámbitos de la Educación Física (EF) y del deporte, de la recreación y de la rehabilitación, por lo tanto se constituye en un conjunto de actividades que le permitan a la persona no sólo mejorar sus capacidades físicas, sino también mejorar las interrelaciones sociales. Las diferentes formas de movimiento, no sólo son necesarias en aquellas personas dotadas de desarrollo motor dentro de los parámetros "normales", por el contrario, en cualquier condición que la persona se encuentre precisa moverse para continuar y sentirse vivo. Fonseca (1998) explica que desde la exteriorización de las emociones, hasta la conciencia y la individualización del propio cuerpo, el movimiento es revelador de una conquista progresiva de la independencia, que esto último para las personas con discapacidad es fundamental.

Cuando se habla de actividad motora adaptada no se está haciendo referencia a que la actividad sea exclusiva para personas con discapacidad, ni que adaptar signifique facilitar. En esta línea, Rodrigues (2006, p. 41) expresa que adaptar es adecuar la exigencia de la tarea al nivel de desempeño del alumno. El autor agrega que, cada vez que se altera la exigencia y las condiciones de desempeño de una actividad, de manera que un determinado alumno pueda realizarla o envolverse en un proceso de aprendizaje, se está adaptando. Además, "[...] tornar la actividad más exigente en términos perceptivos, conquista de decisiones, desempeño motor o elaboración cognitiva es también adaptar".

En este artículo se realiza un análisis descriptivo de los últimos tres planes de estudios del Centro de Montevideo - ISEF (1992, 1996 , 2004) con relación al eje actividad motora adaptada, denominada generalmente en nuestro país EF adaptada para personas con discapacidad. El artículo se estructuró en tres apartados: en el 
primero se realiza una descripción de los programas estudiados con relación al eje actividad motora adaptada; en el segundo, se analiza la formación que existe en el área de la discapacidad en los planes de estudios analizados y en el tercero, se describe el modelo médico de la discapacidad y su influencia en el diseño de los programas.

Para el análisis de los planes de estudios se consideraron los siguientes aspectos:

*denominación,

*cantidad de asignaturas relacionadas con contenidos de la actividad motora adaptada en cada plan,

*carga horaria de las asignaturas vinculadas a la actividad motora adaptada,

*nivel en el que se encuentra en el plan de estudios,

*carácter de la misma: del tronco común, del perfil complementario de centro, disciplina complementaria de libre curso, obligatoria, optativa,

*contenidos.

Fundamentalmente, el último punto sumado a la fundamentación y los objetivos de los programas serán analizados a la luz de las concepciones de discapacidad existentes y de los modelos de discapacidad.

\section{ANÁLISIS DESCRIPTIVO DE LAS ASIGNATURAS VINCULADAS A LA ACTIVIDAD MOTORA ADAPTADA DE LOS PLANES DE ESTUDIOS DE 1992, 1996 Y 2004}

En la tabla 1 se presenta la denominación de las asignaturas pertenecientes al eje actividad motora adaptada en cada uno de los planes de estudio analizados, cantidad de asignaturas, carga horaria, nivel en el que se encuentran, el carácter de las mismas y los contenidos. 
Cuadro 1: Características de las asignaturas vinculadas a la actividad motora adaptada en los tres planes de estudio.

\begin{tabular}{|c|c|c|c|c|c|}
\hline & 1992 y 1996 & \multicolumn{3}{|c|}{1992 - Orientación excepcionales } & 2004 \\
\hline Denominación & $\begin{array}{l}\text { La EF en la } \\
\text { persona con } \\
\text { discapacidad }\end{array}$ & $\begin{array}{l}\text { Psicología } \\
\text { diferencial }\end{array}$ & Teprade IV & $\begin{array}{l}\text { Introducción a la } \\
\text { fisiopatología }\end{array}$ & $\begin{array}{c}\text { EF adaptada para } \\
\text { personas con } \\
\text { discapacidades }\end{array}$ \\
\hline Carga horaria & 48 semestrales & \multicolumn{2}{|c|}{48 semestrales } & 32 semestrales & 60 semestrales \\
\hline $\begin{array}{l}\text { Cantidad de } \\
\text { asignaturas }\end{array}$ & 1 & \multicolumn{3}{|c|}{3} & 1 \\
\hline Nivel & $\begin{array}{l}\text { Tercer nivel - } \\
3^{\text {er }} \text { semestre }\end{array}$ & \multicolumn{3}{|c|}{ Cuarto nivel $-4^{\text {to }}$ semestre } & $\begin{array}{l}\text { Cuarto nivel - } \\
2^{\text {do }} \text { semestre }\end{array}$ \\
\hline Carácter & $\begin{array}{c}\text { Tronco común - } \\
\text { Obligatoria }\end{array}$ & \multicolumn{3}{|c|}{ Orientación - Optativo } & $\begin{array}{c}\text { Perfil } \\
\text { complementario } \\
\text { de centro - } \\
\text { Optativa }\end{array}$ \\
\hline Contenidos & $\begin{array}{l}\text { 1- La persona con } \\
\text { discapacidad. } \\
\text { 2- Las diferentes } \\
\text { discapacidades. } \\
\text { 3-El profesor de EF } \\
\text { y el discapacitado. }\end{array}$ & $\begin{array}{c}\text { 1- Aspectos } \\
\text { generales de la } \\
\text { psicopatología. } \\
\text { 2- Los grandes } \\
\text { cuadros } \\
\text { psicopatológicos } \\
\text { 3- Las } \\
\text { discapacidades. } \\
\text { 4- El niño } \\
\text { hiperactivo. } \\
\text { 5-El autismo. } \\
\text { 6-El entorno } \\
\text { social del } \\
\text { discapacitado } \\
\text { (equipo de } \\
\text { trabajo, padres). }\end{array}$ & $\begin{array}{l}\text { 1- Recreación. } \\
\text { 2- Excepcionales. } \\
\text { 3- Investigación. }\end{array}$ & $\begin{array}{l}\text { 1- Patol ogía neuro } \\
\text { psiquiátrica. } \\
\text { 2- Patología } \\
\text { metabólica. } \\
\text { 3- Patología } \\
\text { reumatológica. } \\
\text { 4- Patología } \\
\text { cardiorrespiratoria. }\end{array}$ & $\begin{array}{c}\text { 1- Aspectos } \\
\text { introductorios de la } \\
\text { EF adaptada para } \\
\text { personas con } \\
\text { discapacidad. } \\
\text { 2- Conceptos } \\
\text { generales de las } \\
\text { diferentes } \\
\text { discapacidades. } \\
\text { 3- Estimulación } \\
\text { precoz en las } \\
\text { discapacidades. } \\
\text { 4- Juegos y deportes } \\
\text { adaptados. } \\
\text { 5- La importancia } \\
\text { del equipo } \\
\text { multidisciplinario. } \\
\text { 6- Integración e } \\
\text { inclusión social de } \\
\text { las personas con } \\
\text { discapacidad a } \\
\text { través de la EF } \\
\text { adaptada. }\end{array}$ \\
\hline
\end{tabular}

Fuente: Elaboración propia (2011).

${ }^{1}$ Teoría y práctica de la didáctica especial.

¿La información que se presenta en los cuadros, fue extraída de los programas mayormente en forma textual; algunas veces fue necesario realizar recortes eliminando información no pertinente para los objetivos de este estudio.

Movimento, Porto Alegre, v. 18, n. 03, p. 203-218, jul/set de 2012. 
El eje actividad motora adaptada estaba presente en el plan de estudios que se implantó a partir de 1992 a través de una asignatura obligatoria de carácter troncal y de un trayecto u orientación optativa. Antes de esa fecha, en el plan de estudios anterior (1981) no existía ninguna asignatura vinculada al área de la discapacidad.

De la tabla se desprende que, en el plan de estudios de 1996 se mantiene una asignatura orientada al área de la discapacidad, la cual tiene exactamente las mismas características que en el plan de 1992, conservándose de esta manera un primer acercamiento de los futuros docentes al área. Sin embargo, la orientación denominada "excepcionales" fue eliminada, quedando las dos restantes: orientación deportes y orientación recreación.

Por otra parte, el plan de estudios del año 2004 contempla una asignatura vinculada al área de la discapacidad, con una carga horaria de 60 horas semestrales y que es cursada en forma optativa por los estudiantes ya que pertenece al perfil complementario de centro ${ }^{3}$. De esta descripción se desprenden dos aspectos interesantes: por un lado el aumento de la carga horaria con relación al plan de estudios de 1996 (doce horas de incremento) y por otro, en el plan 2004 se constituye como una asignatura de perfil complementario de centro y por lo tanto los estudiantes tienen la posibilidad de cursarla si es que lo desean de acuerdo al trayecto curricular que quieran realizar en su formación de grado. De esta manera, todos aquellos estudiantes que no eligen cursar la asignatura, culminan sus estudios en la Licenciatura sin tener una formación introductoria en el área de la actividad motora adaptada. Situación semejante plantea Sosa (2010) en un artículo que aborda la formación profesional en EF sobre discapacidad, al analizar los planes de estudio de los cursos de graduación de cinco universidades argentinas. La autora encontró una introducción a la temática de la discapacidad de carácter optativo en algunos casos y en otros obligatorio.

\footnotetext{
${ }^{3}$ Los perfiles complementarios se desarrollan desde el primer año de la carrera, facilitando la formación en áreas relacionadas a la EF, el Deporte y la Recreación desde una óptica de complementación, acorde a las particularidades de cada región del país en donde se ejercerá la profesión (ISEF - URUGUAY, 2004).
} 
La denominación utilizada para nombrar las asignaturas (la EF en la persona con discapacidad y EF adaptada para personas con discapacidad) no difiere tanto de la utilizada en los cursos de graduación en Brasil, donde son llamados EF especial, EF adaptada y actualmente actividad motora adaptada (RIBEIRO; ARAÚJO, 2004). Sin embargo, varía considerablemente con la terminología utilizada para nombrar las asignaturas en Argentina (Estrategias de abordaje en sujetos con necesidades educativas especiales; Didáctica para la integración en EF; Introducción a la actividad física especial; Pedagogía crítica de las diferencias y atención a la diversidad) (SOSA, 2010).

Con relación a las tres asignaturas que componen la orientación "excepcionales", se hace necesario detenerse en la terminología empleada para denominar la orientación. "Excepcional" significa "[...] que se aparta de lo ordinario, o que ocurre rara vez" (REAL,...2006, p. 1015). Con esta terminología se hace referencia a que la persona con discapacidad es "[...] diferente a la norma" o "[...] sale de lo normal". A su vez, los nombres de dos de las tres asignaturas también contienen palabras que estigmatizan. "diferencial" hace referencia a "perteneciente o relativo a la diferencia de las cosas" (REAL..., 2006, p. 822) y "fisiopatología" al "estudio de la relación entre las funciones del organismo y sus posibles alteraciones" (REAL..., 2006, p. 1063). Además, la "palabra "patología" es definida como la "parte de la medicina que estudia las enfermedades [...] conjunto de síntomas de una enfermedad" (REAL..., 2006, p. 1702).

\section{LA FORMACIÓN EN ACTIVIDAD MOTORA ADAPTADA}

De acuerdo a Rodrigues (2006), la actividad motora adaptada representa hoy en día un campo profesional, científico y un área de formación. Por su parte Diehl (2003) apunta que la misma surge en el ámbito académico a nivel mundial en la década del '70, considerándose una de las áreas de más reciente profesionalización en Brasil y tal vez una de las adquisiciones más significativas de las universidades brasileras en el campo de la EF. La autora explica 
que específicamente en Brasil: "[...] la discusión sobre este tema tuvo su inicio en el medio universitario en las décadas de 1980 y 1990 en las universidades públicas y particulares" (DIEHL, 2008, p. 36). Ribeiro y Araújo (2004) agregan que específicamente fue en el año 1992, cuando en los cursos de graduación se incorporaron asignaturas que abordaban contenidos volcados a la atención de las personas con discapacidad. A su vez, los autores resaltan que los cambios curriculares ocurridos en las universidades brasileras "[...] son el resultado de un movimiento social amplio, que no concebía al hombre solo desde una perspectiva física; en consecuencia la EF, necesitaba ser capaz de contemplar todos los aspectos necesarios en la formación de los sujetos" (RIBEIRO; ARAÚJO, 2004, p. 62). En este sentido, el ISEF, como institución educativa de carácter terciario como era en ese entonces, incorporó en su plan de estudios contenidos del área de la discapacidad en los años '90 al igual que muchas universidades brasileras demostrando un incipiente interés en el área ${ }^{4}$.

DiehI (2008), afirma que con la implementación de la actividad motora adaptada en las universidades, el estudiante comenzó a recibir capacitación para actuar con personas con discapacidad. Se torna entonces responsable de la planificación y puesta en práctica de actividades que mejoren el repertorio corporal de movimientos de las personas con discapacidad, así como de actividades que favorezcan la inclusión.

Los profesores son considerados actores sociales que tienen su accionar influenciado culturalmente. Expresan en su práctica docente determinados valores y concepciones de acuerdo con los contextos culturales en los que se constituyen, como la preparación profesional a la cual tuvieron acceso, el contexto educativo en que trabajan, entre otros. En este sentido, se entiende como necesario que la formación en el área de la discapacidad tenga un carácter obligatorio y sea asumida por una asignatura (por la especificidad de

${ }^{4}$ Dentro de América del Sur, Brasil al igual que Argentina se constituyen como los países con más avances en torno a la actividad motora adaptada, con docentes reconocidos a nivel internacional, publicaciones y congresos en el área. 
muchos temas que se abordan) en los cursos de graduación en EF, aunque ello no desestima que tenga un carácter transversal con el resto de las asignaturas que componen un plan de estudios. Al respecto, Sosa (2010) se cuestiona si tiene que existir una asignatura específica en los planes de formación, o si sería más inclusivo trabajar en el abordaje con el eje transversal de la temática en las distintas asignaturas.

Asimismo, en la fundamentación y objetivos de las asignaturas en el área de la discapacidad de los tres planes de estudios analizados, se hace hincapié en la necesidad de que el estudiante se familiarice con el área indicando, de esta manera, la importancia de tener una formación básica para todos los estudiantes durante la Licenciatura. Un ejemplo de ello es lo que se encuentra plasmado en la asignatura del plan de estudios de 1992 y 1996: "dado el espacio que la persona discapacitada ${ }^{5}$ tiene en el ámbito social, se hace necesario contemplar en el currículo del I.S.E.F. un acercamiento básico a esta área [...]" (ISEF - URUGUAY, 1996, p. 60).

Con relación a los objetivos, se observa que en el plan de 2004 se pretende que el estudiante adquiera competencias específicas en relación al área de la discapacidad, así como "propiciar espacios de reflexión crítica en torno a la temática de la discapacidad" (ISEF URUGUAY, 2004, p. 1). En los planes de 1992 y 1996 se aspira a "sensibilizar al estudiante en el área de la persona con discapacidad", aproximándolo al conocimiento de las diferentes discapacidades (ISEF - URUGUAY, 1996, p. 187).

\section{EL MODELO MÉDICO DE LA DISCAPACIDAD: SU INFLUENCIA EN EL DISEÑO DE LOS PROGRAMAS}

Con respecto a la terminología empleada para referirse a la persona con discapacidad, del análisis del plan de estudios de 1992 y 1996 se ubican dos palabras que se consideran peyorativas $\mathrm{y}$

\footnotetext{
${ }^{5}$ La terminología aquí utilizada se apoya en un modelo médico de la discapacidad como se analizará más adelante.
} 
estigmatizantes: retardo mental y discapacitado. A ambas se las puede encontrar en la bibliografía de las décadas del '70 y '80; la primera, básicamente hace referencia a la discapacidad intelectual y la segunda implica una forma de denominar a esta población. Por otra parte, en la orientación "excepcionales" del plan de estudios de 1992, se encontraron un sinnúmero de palabras despectivas que son usadas comúnmente para referirse a las personas con deficiencia: diferencial, discapacitado, minusvalía, limitaciones del discapacitado, excepcional.

De acuerdo con Gaio (2006) el concepto de discapacidad genera polémica, encontrando contradicciones generadas por los términos normal, anormal, discapacitado, excepcional. Por otra parte, Jannuzzi (1992) sostiene que cuando se quiere definir a la deficiencia lamentablemente los términos empleados hacen referencia a la "falta de", a la exclusión y a la imperfección, no considerando a la diferencia.

Se comparte la idea de esta autora que se encuentra ejemplificada en uno de los objetivos de la asignatura "Introducción a la fisiopatología" de la orientación excepcionales, donde se plantea "aportar los conocimientos básicos de Patología General para conocer las limitaciones del discapacitado [...]" (ISEF - URUGUAY, 1996, p. 3).

De acuerdo a Lancillotti (2003), el concepto de discapacidad ha ido variando a lo largo de la historia, a medida que las condiciones sociales se modificaron por la propia actividad del hombre, generando nuevas necesidades en relación con su medio social. En este sentido, la Organización Mundial de la Salud (OMS) realizó una modificación en el año 2001 a la Clasificación Internacional de Deficiencia, Discapacidad y Minusvalía (CIDDM), propuesta en 1980.

La nueva Clasificación Internacional del Funcionamiento, de la Discapacidad y de la Salud (CIF) de la OMS (2001), puede ser empleada como herramienta de política social, en la planificación de sistemas de seguridad social, sistemas de compensación y para diseñar e implementar estrategias. En esta clasificación el funcionamiento y la discapacidad de un sujeto son vistas como una "interacción dinámica entre los estados de salud (enfermedades, trastornos, lesiones, 
traumas, etc.) y los factores contextuales [...]" (OMS, 2001, p. 9). Dentro de éstos, se incluyen los factores personales y los ambientales. La discapacidad aquí engloba "[...] a las deficiencias que refieren a las lesiones o ausencia de funciones o estructuras corporales, a las limitaciones en las actividades que puede presentar una persona en su vida cotidiana y a las restricciones en la participación social" (BAGNATO, 2007, p. 10). Además, Pappous (2007, p. 52) explica que la nueva clasificación "pone de manifiesto que la discapacidad no se define en sí, sino que se refiere también a las relaciones que el individuo mantiene con la sociedad en la cual vive y en las condiciones ambientales".

Para explicar la clasificación de discapacidad y funcionamiento se propusieron dos modelos: el médico y el social. "La CIF está basada en la integración de estos dos modelos opuestos. Con el fin de conseguir la integración de las diferentes dimensiones del funcionamiento la clasificación utiliza un enfoque biopsicosocial" (OMS, 2001, p. 22).

En las asignaturas del eje actividad motora adaptada de los planes de estudios del año 1992 y 1996, incluyendo el trayecto, no se contempla en los contenidos a enseñar la Clasificación Internacional de Deficiencia, Discapacidad y Minusvalía, pero sí es tenida en cuenta en el plan de estudios de 2004 (en la unidad 1). Sin embargo, en este último plan no se presenta en los contenidos la nueva Clasificación Internacional del Funcionamiento, de la Discapacidad y de la Salud, aprobada en el año 2001 por la OMS. A pesar de esto último, en el programa de la asignatura (la EF adaptada para personas con discapacidad) del plan 2004, se contempla una unidad temática volcada a la integración e inclusión social de las personas con discapacidad, lo cual pone de manifiesto la importancia que se le da al contexto (aspecto considerado en la CIF), en este caso para promover la inclusión.

Con el objetivo de profundizar en los modelos de discapacidad y su influencia en los diseños de los programas se describen ambos y se los relaciona con las asignaturas estudiadas en este artículo. El modelo médico "extendido desde principios del siglo veinte, ponía el 
acento en la "anormalidad" corporal, en la dimensión clínica, el desorden y el grado de "discapacidad" o deficiencia funcional provocado por la deficiencia" (PAPPOUS, 2007, p. 50).

Esto se puede observar en los contenidos que se plantean en dos de las asignaturas de la orientación "excepcionales". Las asignaturas "Psicología diferencial" e "Introducción a la fisiopatología" están enfocadas a describir básicamente las distintas deficiencias ${ }^{6}$, ejemplo de ello son las unidades temáticas que se escriben a continuación: aspectos generales de la psicopatología; grandes cuadros psicopatológicos; las discapacidades; el niño hiperactivo; el autismo; síndromes piramidales y extrapiramidales; convulsiones; diabetes mellitus; insuficiencia cardíaca, otros. Con esto, no se está afirmando que la descripción de las discapacidades no sea importante, pero se torna fundamental que la misma esté relacionada al estudio del movimiento humano, área de actuación del profesor de EF. Sobre este asunto, Sosa (2010, p. 15) expresa que en los programas estudiados "pareciera que no se puede prescindir al hablar de discapacidad, de nombrar las diferentes clasificaciones de la deficiencia [...] éstos ponen acento en la enseñanza de descripción de distintas patologías [...]". Mauerberg-deCastro (2005), agrega que el modelo médico tuvo influencia en todas las profesiones asociadas a la asistencia, intervención y salud en general.

Por su parte, el modelo social, considera la discapacidad fundamentalmente como una cuestión de origen social y como un asunto centrado en la completa integración del sujeto en la sociedad. En este modelo, la discapacidad no es netamente personal, sino un problema que requiere la acción social de todos sus miembros para realizar las adaptaciones necesarias que posibiliten la completa inclusión de las personas. La principal idea de este modelo radica en que es la sociedad la que "discapacita" a las personas con discapacidad, por lo tanto no coloca a la discapacidad en el sujeto con deficiencia sino en el contexto en el cual la persona se encuentra inmersa (PAPPOUS, 2007).

${ }^{6}$ La deficiencia es toda pérdida o anormalidad de una estructura o función piscológica, fisiológica o anatómica (OMS, 1983). 
La fundamentación escrita en el programa de la asignatura "EF en la persona con discapacidad" del plan 1992 y 1996, deja entrever que subyace el modelo social de la discapacidad, sin embargo, la terminología utilizada a lo largo del programa y los contenidos subdivididos en tres unidades temáticas (las diferentes discapacidades, características psíquicas, físicas y sociales de las discapacidades, el profesor de EF y el discapacitado, etc.) no mantienen coherencia con este modelo.

Continuando con el análisis, la asignatura del eje actividad motora adaptada del plan de estudio de 2004 intenta apoyarse en este modelo de discapacidad para plantear su fundamentación, objetivos y contenidos. En este sentido, en la fundamentación se explicita que:

[...] desde hace algunos años, la discusión acerca de una sociedad inclusiva y no exclusiva con relación a las personas con discapacidad se sucede en diferentes ámbitos y desde diferentes perspectivas: educacional, médica, psicológica y social visualizando la realidad desde diferentes miradas, muchas de ellas alejadas de la visión de la propia persona con discapacidad (ISEF - URUGUAY, 2004, p. 1).

Asimismo, algunos contenidos se abordan desde un modelo médico de la discapacidad y otros bajo un modelo tendiente a lo social.

Por último, en la asignatura Teprade IV que forma parte de la orientación, integra en los contenidos dentro de lo que denomina población excepcional a las personas con discapacidad, a los niños de edad inicial y a los adultos mayores. Cabría preguntarse cuál es el fundamento para considerar a los dos últimos grupos dentro de la población con discapacidad, aunque Ribeiro y Araújo (2004) explican, que muchas veces los adultos mayores por las alteraciones mecánicas en los patrones motores que presentan, se encuadran dentro de la población con discapacidad. 


\section{Consideraciones finAles}

A lo largo del presente artículo, se fundamentó la importancia de que exista una formación básica y obligatoria en actividad motora adaptada, sin embargo la misma, no debería contemplar solamente los aspectos médicos de las discapacidades. De nada sirve, diseñar un trayecto de orientación relacionado a la discapacidad, si los contenidos que allí se abordan están empapados de nociones de "normalidad" y "anormalidad". De hecho, las características de las distintas deficiencias deben estar atravesadas por las posibilidades que tienen las personas con discapacidad de envolverse en una variedad de prácticas corporales adaptadas, colocando la mirada en las posibilidades y no en las limitaciones.

Se cree que para plantear objetivos, planificar actividades, pensar en recursos metodológicos, adaptar propuestas, el profesor de EF tiene que conocer en primer término en qué consiste la deficiencia de la persona; se recalca la idea de que no se puede planificar en esta área sin tener conocimientos técnicos de las discapacidades y de las posibilidades de movimiento en función de la discapacidad y de la propia persona. En este sentido entonces, por un lado, se torna fundamental que se contemple una formación obligatoria para todos los estudiantes y por otro, que la misma contemple la nueva conceptualización de discapacidad de la CIF donde se considera a la persona y su interrelación en variados contextos.

A modo de cierre, se comparte la idea de Costa y Duarte (2006), con relación a que la actividad física es fundamental en las personas con discapacidad, por la significativa posibilidad que puede ofrecer en la búsqueda de un sentido para su vida. Pero, si se quiere contribuir desde la EF, es necesario preparar a los futuros docentes en los cursos de graduación, que ayuden a promover espacios para la práctica de actividad motora adaptada, cambiando de a poco la realidad existente que muestra escasez de posibilidades de movimiento para las personas con discapacidad. 
A formação dos professores de Educação Física na área da deficiência: uma analise dos planos de estudo de 1992, 1996 e 2004 do curso de professores do ISEF - Montevidéu

Resumo: No artigo se apresentam aspectos vinculados à formação na área da atividade motora adaptada dos professores de Educação Física a partir da análise das disciplinas relacionadas a essa área nos três últimos planos de estudo (1992, 1996 e 2004) do Curso de Professores do Instituto Superior de Educação Física (Montevidéu). Os resultados obtidos mostram um interesse em oferecer pelo menos uma disciplina vinculada á área da deficiência, no entanto embora se possa observar uma mudança para o modelo social no plano de 2004, observa-se que a fundamentação, objetivos e conteúdos se apoiam no modelo médico da deficiência vigente ainda hoje.

Palavras-chave: docentes. Deficiência. Cursos de capacitação. Currículo.

The preparation of Physical Education teachers in the area of disability: an analysis of the 1992, 1996 and 2004 curriculae of the teachers' training course at THE ISEF - Montevideo

Abstract: This article elucidates aspects which relate to the preparation process of Physical Education teachers in the area of adapted motor activity, based on the study of subjects pertaining to the area in the three latest curriculae (1992, 1996 and 2004) of the Teachers' Training Course of the Instituto Superior de Educación Física. The results achieved reveal an interest in offering at least one subject involved in the area. Notwithstanding, its principles, objectives and contents are framed in a medical pattern of disability, though it is to be noted that some change towards a social pattern appears in the 2004 curricula.

Keywords: Faculty. Disability. Training Courses. Curriculum. 


\section{REFERENCIAS}

BAGNATO, María José. Inclusión social de personas con discapacidad en las instituciones de educación terciaria y universitaria: un aporte desde la Universidad de la República. Comisión Sectorial de Extensión y Actividades en el Medio. Montevideo. 2007. Disponible en: <http://www.rau.edu.uy/fcs/dts/Gedis/ proyectoinclusionsocial2007.pdf> Acceso el: 30 mar. 2010. 27 p.

COSTA, Alberto Martins da; DUARTE, Édison. Atividade física, saúde e qualidade de vida das pessoas com deficiência. In: RODRIGUES, David (Org.). Atividade motora adaptada: a alegria do corpo. São Paulo: Artes Médicas, 2006. p. 120129.

GAIO, Roberta. Para além do corpo deficiente. Histórias de vida. Jundaí: Fontoura, 2006.

DIEHL, Rosilene Moraes. Atividade motora adaptada. In: ENCONTRO NACIONAL DE PROFISSONAIS DE EDUCAÇÃO FÍSICA, 29, 2003, Capão da Canoa. Anais. Capão da Canoa, 2003.

DIEHL, Rosilene Moraes. Jogando com as diferenças. Jogos para crianças e jovens com deficiência: em situação de inclusão e em grupos específicos. 2. ed. São Paulo: Phorte, 2008.

FONSECA, Vitor da. Da filogênese à ontogênese da motricidade. Porto Alegre: Artes Médicas, 1998.

ISEF. Anteproyecto Diseño Curricular 2004. Montevideo: Instituto Superior de Educación Física, 2003.

ISEF. Diseño curricular plan 1992: curso de profesores Montevideo: Instituto Superior de Educación Física., 1996.

ISEF. Reglamento plan de estudios 2004: curso de Profesores de Educación Física. Montevideo: Instituto Superior de Educación Física., 2004.

JANUZZI, Gilberta. A luta pela Educação do Deficiente Mental no Brasil. 2. ed. Campinas: Editora Autores Associados, 1992.

LANCILLOTTI, Samira Saad Pulchério. Deficiência e trabalho: redimensionando o singular no contexto universal. Campinas: Autores Associados, 2003.

MAURBERG-DECASTRO, Eliane. Atividade Física adaptada. Riberão Preto: Tecmedd, 2005.

OMS. Clasificación internacional de deficiencias, discapacidades y minusvalías. Madrid: Inserso, 1983. 
OMS. Clasificación internacional del funcionamiento de la discapacidad y de la salud: CIF Ministerio de trabajo y asuntos sociales. Madrid. 2001. Disponible en: <http://ccp.ucr.ac.cr/bvp/pdf/desarrollohumano/oms-clasificacion-01.pdf> Acceso en: 20 mar. 2010. 318 p.

PAPPOUS, Athanasios. Inclusión e integración en Europa: Hacia una educación Física y deportiva adaptada. In: EUSSE CASTRO, Edgar Danilo (Ed.). Educación física y discapacidad: prácticas corporales inclusivas. Medellín: Funámbulos editores, 2007. p. 49-62.

REAL ACADEMIA ESPAÑOLA. Diccionario de la lengua española. 21. ed. Buenos Aires: Espasa, 2006. 2 v.

RIBEIRO, Sonia Maria; ARAÚJO, Paulo Ferreira de. A formação acadêmica refletindo na expansão do desporto adaptado: uma abordagem brasileira. Revista Brasileira de Ciências do Esporte, Campinas, v. 25, n. 3, p. 57-69, maio 2004.

RODRIGUES, David. As dimensões de adaptação de atividades motoras. In:

(Org.). Atividade motora adaptada: a alegria do corpo. São Paulo: Artes Médicas, 2006. p. 40-47.

SOSA, Laura. La formación profesional en Educación Física sobre discapacidad. Educación Física Argenmex: temas y posiciones, La Plata, 2010. Disponible en: <http://www.argenmex.fahce.unip.edu.ar> Acceso en: 15 feb. 2011.

Dirección de correo:

Calle Gabriel Pereira 2845 Apto 502

C.P. 11300

Montevideo

Uruguay

Recebido em: 27.04.2012

Aprovado em: 16.07.2012 
